\title{
Why do we live in a 4D world: Can cosmology, black holes and branes give an answer?
}

\author{
Konstantin G. Zloshchastiev \\ Instituto de Ciencias Nucleares, Universidad Nacional Autónoma de México, A.P. 70-543, México D.F. 04510, México
}

\begin{abstract}
We derive the general form of the cosmological scalar field potential which is compatible both with the existence of black holes and p-branes related to string/M theory and with multidimensional inflationary cosmology. It is shown that the scalar potential alters non-trivially from dimension to dimension yet always obeys one single equation where the number of spacetime dimensions is a free parameter. Using this equation we formulate an eigenvalue problem for the dimensionality parameter. It turns out that in the low-energy regime of sub-Planckian values of the inflaton field, i.e., when the Universe has cooled and expanded sufficiently enough, the value four arises as the largest admissible (eigen)value of this parameter.
\end{abstract}

PACS numbers: 11.25.-w, 98.80.Cq, 04.70.Bw

Modern trends in high-energy physics such as string/M theory suggest that the unification of all fundamental interactions is impossible without increasing the number of actual spacetime dimensions, $D$, from 4 to 10 or even 11 . It seems that only in higher dimensions one can reconcile general relativity with quantum mechanics and construct a self-consistent theory. Nevertheless, despite the continuously growing popularity and successes of higherdimensional models one should not forget one of the fundamental problems of string/M theory: to explain why at low energies our world is effectively four-dimensional to such a high degree of precision.

Recent efforts to dynamically derive or explain $4 \mathrm{D}$ spacetime have been made in the matrix formulation of type IIB superstrings but no definite conclusion has been made so far [1, 2, 3]. Also, attempts have been made in alternative theories of quantum gravity, such as Causal Quantum Gravity [4] but that theory has too little in common with M theory.

Other attempts were also made in several multidimensional models imposing one or another predefined way of splitting a higher-dimensional manifold into the external space (the observable Universe) and an internal one. For instance, working in 11D supergravity quantum cosmology if one assumes the seed instanton of the Universe to be a product of two spheres, then it can be proven that the external spacetime must be 4D [5]. Although, in such models the problem was not entirely solved but rather reduced to the well-known problem of which way of dimensional reduction the Universe uses to descend from 11D to $4 \mathrm{D}$. This is still far from being answered.

Thus, the problem of formation of the Universe's dimensionality still persists. Is it possible to solve it in a way consistent with $M$ theory ideas? In this paper we will try to get an affirmative answer.

In fact, as compared to all other parameters which appear in field theory, the parameter $D$ has a number of distinctive features which, on one hand, create difficulties with solving the above-mentioned problem but, on the other hand, hint about possible clues. First, we know that any field theory in spacetime is defined by virtue of the action functional, $S[\psi(x)]=\int L\left(\psi_{i}, \partial \psi_{i}\right) d^{D} x$, where $\psi_{i}$ is a set of the fields built into the theory, $L$ is the Lagrangian function. Here the spacetime dimensionality $D$ is involved both in the integration measure of the functional, $d^{D} x$, and in the notion of distance between events in spacetime, thus it is the parameter which must be fixed before one starts specifying the Lagrangian itself. Therefore, $D$ can not be directly derived from a theory which acts in spacetime. Second, being an integer rather than a continuous parameter, $D$ hints about the possibility that it could be a discrete eigenvalue of a certain dynamical theory (which acts not in spacetime, according to the aforesaid).

Below we show that both suppositions are indeed very close to the truth. We demonstrate that the values of the parameter $D$ are dynamically derivable from a differential equation defined in a certain space (called the coupling space) whereas the properties of the latter are determined by black hole physics closely related to $\mathrm{M}$ theory, supersymmetry (SUSY) and p-branes.

We start by recalling that the global dynamics of the Universe is determined by long-range fields. From the Standard Model we know that Yang-Mills and fermionic fields are short-range thus here we can restrict ourselves to the Abelian sector. The latter includes gravity described by the Lorentzian metric tensor $g_{M N}$ (capital Latin indices run from 1 to $D$ ), real scalar field $\phi$ (often called also the inflaton as it has been widely used in cosmology in scalar-driven inflationary models) and several gauge fields described by antisymmetric tensors of different ranks. Thus, our action functional must contain at least the graviton and the inflaton. To this we add also a couple of gauge fields: the electromagnetic one described by the antisymmetric tensor of second rank, $F_{M N}$, and the one of rank $\tilde{p}$, with the components $F_{(\tilde{p}) M \ldots N}$. The gauge fields are added just to check whether they affect the problem we are going to study. The simplest effective action one can thus write is (we work in the Einstein frame):

$S=\int d^{D} x \sqrt{-g}\left[R-\frac{1}{2}(\partial \phi)^{2}+\Xi(\phi) F^{2}+\Psi(\phi) F_{(\tilde{p})}^{2}-V(\phi)\right]$,

where $(\partial \phi)^{2}=\partial_{M} \phi \partial^{M} \phi, F^{2}=F_{M N} F^{M N}, F_{(\tilde{p})}^{2}=$ $F_{(\tilde{p}) M \ldots N} F_{(\tilde{p})} M \ldots N$, and $g=\operatorname{det}\left|g_{M N}\right|$ and $R$ is the scalar curvature constructed out of $g_{M N}$. By this action we mean an ensemble of theories which are equivalent with respect to their dynamical content but act in different $D$. Then we can regard $D$ as a free parameter from now on. For further, it will be convenient to assume $D \equiv \tilde{p}+2$ and work in terms of the parameter $\tilde{p}$. 
The explicit values of the Maxwell-scalar coupling $\Xi$, the $\tilde{p}$-tensor-scalar coupling $\Psi$ and the scalar selfcoupling (potential) $V$ are largely unknown, even in string/M theory [6], except in the case where they were obtained in the tree-level superstring approximation [7] but those were too simple to describe any realistic phenomena because SUSY gets broken at early stages of the evolution of the Universe. Thus, our task now is to find the coupling functions without using the supersymmetrical arguments solely but engaging instead some other, physically more general, arguments.

The first thing we require from the theory (11) is that it should not forbid black hole solutions at any physically relevant $D$ (apart from being compatible with inflation and string/M theory, of course). Indeed, the mathematical absence of black hole solutions would lead to a loss of protection of a theory from naked singularities [8] and thus to undesirable violations of the Cosmic Censorship principle [9, 10, 11]. This is especially dangerous in our case: if, according to modern cosmological views, the Universe began as an extremely hot, dense and compact higher-dimensional object then the ubiquitous fluctuations of the spacetime metric which took place at that stage, inevitably led to the appearance of spacetime singularities. As long as it is impossible to set initial data in a singular point, the evolution of physical objects inside the spatial domains which are causally-dependent from singularities is essentially unpredictable. Then during the inflationary era such regions would become extremely large. Black holes protect from this by "dressing" the singularities with event horizons and thus causally disconnecting them from the rest of the Universe. Besides, there exist some arguments that the existence of black holes places an upper bound for the cosmological constant parameter responsible for the rate of expansion of the Universe and thus prevents the latter from hyperaccelerated inflation [12].

In view of the aforesaid one may wonder how could it be that black holes, having such a small size and mass comparing to those of the Universe, can nevertheless influence its global properties? One should recall, however, that the notion of event horizon is a non-local one - in general it requires the knowledge of not only the distribution of matter in space but also its future evolution, and depends on the fate of Universe 13. Thus, the existence of event horizons inevitably affects the large-scale structure of the whole space. In the case of scalar-driven inflationary cosmology black holes act with the aid of the global scalar field - it is well-known that the scalar "nohair" theorems forbid the appearance of black holes for a large set of scalar potentials, e.g., convex or positive semi-definite 14]. Thus, by far not every inflaton potential, and hence by far not every inflationary scenario, is compatible with the existence of regular horizons (here we call a horizon regular if not only the metric but also other fields do not become singular on it).

Further, in turn black holes need for a proper description of their own properties (microstates, entropy, etc.) certain type of M-theory solutions - branes [15]. Therefore, the mathematical absence of brane-like solutions in a theory would cause, apart from incompatibility with the main idea of $\mathrm{M}$ theory, serious difficulties with a consistent microscopical description of such phenomena as the black hole thermodynamical laws. Also, speaking more generally, the branes as extended objects are natural generalizations of the notion of a point particle and thus can serve as a better approximation to the quantummechanical reality: for instance, one can ask a question to what extent the entangled collective quantum states (e.g., the Cooper pairs) which act as a whole can be regarded as (a plain set of) point-like objects and is there any better effective description of them.

Lastly, to ensure good ultraviolet behaviour we restrict ourselves to the class of those p-branes which are explicitly related to $\mathrm{M}$ theory and tend to supersymmetric BPS states in some limit 16]. We demand thus that our model (11) should be compatible with such branes at any physically admissible $D$. We accentuate that this is a necessary but, of course, not a sufficient condition for a theory to be physically relevant. Nevertheless, we will see straight away that it significantly decreases the number of allowed coupling functions.

Imposing this requirement for the 0-branes from the above-mentioned class (we are looking for necessary conditions, therefore, we can take 0-branes for simplicity), using the corresponding ansatz from ref. 17] and applying the approach of ref. [16] assuming $D$ arbitrary, we obtain that the most general couplings of (11) which allow such 0-branes are those obeying the following secondorder differential equations (see the Appendix for more details):

$$
\begin{aligned}
& \hat{\Xi}^{\prime \prime}(\phi)+\frac{4 \eta}{\phi_{1}} \hat{\Xi}^{\prime}(\phi)-\frac{2(\tilde{p}-1)}{\tilde{p}} \hat{\Xi}(\phi)=0 \\
& V^{\prime \prime}(\phi)-\frac{\tilde{p} \operatorname{coth}\left(\frac{\phi}{\phi_{1}}\right)-\eta(\tilde{p}-2)}{\phi_{1}(\tilde{p}-1) / 2} V^{\prime}(\phi)+\frac{2}{\tilde{p}} V(\phi)=0,(3)
\end{aligned}
$$

where $\hat{\Xi}(\phi) \equiv 2 Q^{2} / \Xi(\phi)+\tilde{p} ! P^{2} \Psi(\phi)$, with $Q$ and $P$ being, respectively, the electric and magnetic brane charges (up to a numerical coefficient), and

$$
\phi_{1} \equiv \frac{4 a}{\varsigma \Delta}, \eta \equiv 1-\frac{4(\tilde{p}-1)}{\tilde{p} \Delta}, \Delta=a^{2}+2-\frac{2}{\tilde{p}},
$$

$a$ is some constant parameter related to p-branes 18 (here we assume it free as well), and $\varsigma$ is +1 for elementary (electric) branes and -1 for solitonic (magnetic) ones. The fact that the gauge-scalar couplings $\Xi(\phi)$ and $\Psi(\phi)$ appear in eq. (2) only as the combination $\hat{\Xi}(\phi)$ is explained by the existence of electric-magnetic duality between the 1-form $\mathcal{A}$ (such that $F=d \mathcal{A}$ ) coupled to the worldline of 0 -brane and the $(\tilde{p}-1)$-form $\mathcal{A}_{(\tilde{p}-1)}$ (such that $\left.F_{(\tilde{p})}=d \mathcal{A}_{(\tilde{p}-1)}\right)$.

As long as the solutions of eqs. (2), (3) could be, in general, complex-valued functions with cuts and singularities, the equations must be supplemented with the restriction for the couplings to be physically admissible for real values of the inflaton. Otherwise, the action (11) would become ill-defined from the field-theoretical point of view. We impose thus the following "boundary" condition:

$$
\Xi(\phi), \Psi(\phi), V(\phi) \subset \text { "physical" for all } \phi \in \Re \mathrm{e},
$$


where "physical" means a set of at least real, singlevalued and regular in a finite real domain functions of $\phi$ - as the couplings should be. At first sight, this condition looks too weak to give any physically interesting restrictions. However, it turns out that in some cases it is sufficient to pose a well-defined eigen problem [16].

Further, eq. (2) can be easily solved,

$$
\hat{\Xi}(\phi)=\sigma_{1} e^{\frac{2 \varsigma(\tilde{p}-1)}{a \tilde{p}} \phi}+\sigma_{2} e^{-\varsigma a \phi},
$$

where $\sigma_{i}$ 's are arbitrary integration constants, and it is of no interest to us here, as eq. (4) can not bring any restrictions for the parameter $\tilde{p}$ but only infers that $\sigma$ 's must be real-valued. Thus, it seems that gauge fields, even those having a long range, have little or no influence on the process of the Universe's dimensionality formation.

The other coupling equation, for the scalar field potential, looks more promising in this connection. In fact, it describes the self-interaction of the inflaton which is known to determine the global dynamics of the Universe, according to the scalar-driven inflationary scenarios. Could it thus be that eq. (3) determines not only the potential itself but also the parameter $D$ ?

Due to the complexity of eq. (3) it seems difficult to solve exactly the eigenvalue problem imposed by eq. (4) in the general case. Luckily, one can engage certain physical picture to see things more clearly. Starting from some cosmological epoch, when the characteristic energy became of the order of the string scale, the global dynamics of the Universe can be effectively described by the action (11) at $D>4$ in the supergravity (SUGRA) approximation [19]. At that stage the existence of p-branes did not imply any restrictions for $D$ as the high symmetry ensured that the model (11) has p-brane solutions at any $D$ provided its couplings are compatible with supergravity 7, 18], i. e., the eigenvalues of $D$ have a "continuous" spectrum - in a sense that they take integer values (restricted by $D \leq 11$ so as to exclude the appearance of an infinite number of fields with spins higher than 2) but otherwise arbitrary. However, as the Universe expands and cools, SUSY gets dynamically broken such that in eq. (11) the SUSY-compatible couplings become physically inadmissible (in particular, $V(\phi)$ cannot be set to zero anymore, otherwise inflation cannot start). Therefore, one should go on computing the general couplings directly from eqs. (2) and (3). Those, of course, will include SUGRA couplings as a special case.

All this can be quantitatively visualized if one clocks the history of the early Universe by means of the cosmological scalar field's scale (of course, starting from the epoch when this field has already appeared). Then one could distinguish the following three regimes of eq. (3) and, hence, of $V(\phi)$ :

Top high-energy regime. In the pre- and earlyinflationary Universe nothing could prevent the cosmological scalar from having large initial magnitude. This regime thus corresponds to large values of $\phi$ - such that the cotangent's absolute value in eq. (3) approaches one. Actually, "large" is a way too strong word here: the cotangent's magnitude approaches unity exponentially rapidly so that already at $\left|\phi / \phi_{1}\right| \geq 2$ (in Planck units) it differs from one by less than $4 \%$. The general solution of eq. (3) in this regime is a linear combination of two exponents and no restrictions for $D$ arise from eq. (4). Thus, in this regime the dimensionality parameter is essentially free that confirms aforesaid. Notice that the full supersymmetry can be restored because $V=0$ is also a solution of this equation.

Bottom high-energy regime. As the cosmological time passes, the inflaton's energy density gets diluted by inflation so the scalar starts decreasing its magnitude towards its vacuum value and eventually at some point $\left|\phi / \phi_{1}\right|$ becomes approximately one in Planck units, $\left|\phi / \phi_{1}\right| \approx$ $\left(M_{\text {Planck }}\right)^{\tilde{p} / 2}$. In this regime one should consider eq. (3) in full and study the eigen problem by imposing the condition (4). The dimensionality of spacetime is still above four but perhaps some values of $D$ are already forbidden.

Low-energy regime. The Universe continues expanding so that the scalar gradually approaches its recent value, $\phi \rightarrow \phi_{\text {now }}$, which is very small: $\phi_{\text {now }} \ll\left(M_{\text {Planck }}\right)^{\tilde{p} / 2}$. Therefore, in this regime the cotangent predominates the constant $\eta$-term in eq. (3) so that the general solution can be expressed in terms of the associated Legendre functions 20]:

$$
V(\phi)_{|\phi| \phi_{1} \mid \ll 1}=\left(z^{2}-1\right)^{\frac{\tilde{p}}{2(1-\tilde{p})}}\left(C_{1} P_{\nu}^{\mu}(z)+C_{2} Q_{\nu}^{\mu}(z)\right),
$$

where $z=\operatorname{coth}\left(\phi / \phi_{1}\right)$, the $C_{i}$ 's are arbitrary constants (complex-valued, in general), and

$$
\nu \equiv \frac{1}{1-\tilde{p}}-2, \mu \equiv \frac{1}{\tilde{p}-1} \sqrt{\tilde{p}^{2}-\frac{32 a^{2}(\tilde{p}-1)^{2}}{\tilde{p} \Delta^{2}}} .
$$

Further, the general solution for $V(\phi)_{\left|\phi / \phi_{1}\right| \ll 1}$ is a complex-valued function which has a cut along the real axis for small values of $\left|\phi / \phi_{1}\right|$ (that correspond to large $|z|)$. Therefore, using App. A of ref. [16] one can show that the condition (41) can be satisfied at arbitrary $C_{i}$ (by their proper redefinition) if and only if $\mu$ and $\nu$ both take integer values:

$$
\begin{aligned}
& \nu=n_{\nu}=0, \pm 1, \pm 2, \ldots, \\
& \mu=n_{\mu}=0, \pm 1, \pm 2, \ldots
\end{aligned}
$$

Using the first of these spectral formulae we can draw Table II from which one can see that the maximal allowed eigenvalue of $D$ is 4 . In that case the tensor $F_{(\tilde{p})}$ becomes of a second rank, hence, we arrive at the model previously studied in refs. 12, 16] whereas some of the above-mentioned 0-branes become $4 \mathrm{D}$ black holes with regular scalar and gauge hair. At that, when the gauge field is off, the regular horizon survives only if the parameter $a$ obeys the second of the spectral formulae above (incidentally, eq. (9) brings us to the relation noticed by Hull and Townsend when dealing with extremal black holes in 4D string compactifications [21]). Notice also that in $4 \mathrm{D}$ both spectral formulae become exact, as one can see from eq. (3).

Thus, when the Universe is hot, dense and compact, all the modes of the scalar potential, described by eigenfunctions of $V(\phi)$, are equally permitted. But as the Universe evolves in time, i.e., expands and cools, the potential occupies one of the few (eigen)modes which are allowed by 


\begin{tabular}{cccc}
\hline \hline$n_{\nu}$ & $D-2$ & $n_{\nu}$ & $D-2$ \\
\hline 0 & $1 / 2$ & & \\
1 & $2 / 3$ & -1 & 0 \\
2 & $3 / 4$ & -2 & $\pm \infty$ \\
3 & $4 / 5$ & -3 & 2 \\
4 & $5 / 6$ & -4 & $3 / 2$ \\
5 & $6 / 7$ & -5 & $4 / 3$ \\
$\vdots$ & $\vdots$ & $\vdots$ & $\vdots$ \\
$\infty$ & 1 & $-\infty$ & 1 \\
\hline \hline
\end{tabular}

TABLE I: Correspondence between the spectra of $\nu$ and $\tilde{p}$.

above-mentioned physics. Other modes become dynamically unstable in the low-energy regime. At that, the transition from the "continuous" (i.e., integer but otherwise arbitrary) spectrum of $D$ to $D=4$ happens not continuously but by jumps, due to the discrete nature of $D$.

One can find some similarity between this scenario and the recombination of an electron and a stand-alone hydrogen ion. When the electron has sufficiently high energy (above the ionization threshold), its energy spectrum is essentially continuous. As the energy decreases, e.g., due to the spontaneous emission of photons, the electron gets captured by the ion and its energy enters a discrete spectrum. At that, we know that no description of such binding is possible in terms of classical orbits and trajectories - instead, one should think in terms of quantum superpositions and transition probabilities. One can not exclude the possibility that somewhere a similar uncertainty arises in the very early Universe: there may exist no fully deterministic description of the transition from higher dimensions to $4 \mathrm{D}$ so that the only essential information about this process that one can have in principle is the set of the allowed coupling (eigen)functions, such as $V(\phi)$. However, the analogy of the above-mentioned quantum-mechanical phenomenon with our case should be treated with great care because it is not perfect. In particular, in the quantummechanical case one has a linear superposition of eigenfunctions which correspond to different eigenvalues of energy whereas here we have different regimes of the same function, $V(\phi)$, at different values of its argument. Thus, the interpretation of our eigenproblem is largely an open question so far. It would be interesting to study it in full, i.e., when the inflaton potential stays in the above- mentioned "bottom high-energy" regime. This should provide more details about how the transition from larger values of $D$ proceeds to $D=4$. Besides, if one has the exact solutions of eqs. (2), (3) in hand then using the method described in ref. [17 one can immediately obtain exact generalized 0-brane solutions which are higherdimensional generalizations of black holes.

To conclude, we have determined the self-coupling function (potential) of a cosmological scalar field which would break supersymmetry in such a way as to be compatible with inflationary cosmology and yet preserve some universal features of the low-energy M-theory's solutions. Assuming the number of spacetime dimensions to be a free parameter, we considered the eigenvalue problem for it. It is shown that in the low-energy regime of small values of the cosmological scalar field $D=4$ arises as a largest allowed eigenvalue. Hopefully, knowledge of the behaviour of the inflaton potential at different $D$ will advance also our understanding of other phenomena which took place in the early Universe.

\section{Acknowledgments}

I acknowledge the fruitful discussion about eq. (4) with Hernando Quevedo and Daniel Sudarsky which greatly helped to clarify this point.

\section{APPENDIX: DERIVATION OF THE COUPLING EQUATIONS}

The general 0-brane ansatz can be written in the following form. For metric we assume

$$
\mathrm{d} s^{2}=-e^{U(r)} \mathrm{d} t^{2}+e^{-U(r)} \mathrm{d} r^{2}+e^{A(r)} \mathrm{d} \Omega_{(\tilde{p})}^{2},
$$

where $\mathrm{d} \Omega_{(\tilde{p})}^{2}$ is a metric of a $\tilde{p}$-dimensional $S O$ symmetrical transverse space, and $r$ is the radial coordinate therein. The Maxwell and $\tilde{p}$-form fields are assumed being in the form

$$
F=Q \frac{e^{-\tilde{p} A / 2}}{\Xi(\phi)} \mathrm{d} t \wedge \mathrm{d} r, \quad F_{(\tilde{p}) M \ldots N}=P e^{-\tilde{p} A / 2} \varepsilon_{M \ldots N}
$$

in an orthonormal frame, and $\phi$ is just a function of $r$. From the corresponding equations of motion one can derive the so-called class equation [17]:

$$
\frac{H_{, \phi}}{A_{, \phi}}+\left(\frac{1}{\tilde{p} A_{, \phi}^{2}}+\frac{\tilde{p}-1}{2}\right) H+\frac{e^{A}}{\tilde{p}}\left(\Lambda+e^{-\tilde{p} A} \hat{\Xi}\right)+\tilde{p}=1,
$$

where

$$
H \equiv \frac{1}{\tilde{p}\left(1 / A_{, \phi}\right)_{, \phi}}\left[\tilde{p}(\tilde{p}-1)+e^{A}\left(\Lambda+\frac{\tilde{p}}{2} \Lambda_{,_{\phi}} A_{, \phi}\right)+e^{-(\tilde{p}-1) A}\left(\hat{\Xi}+\frac{\tilde{p}}{2} \hat{\Xi}_{,_{\phi}} A_{, \phi}\right)\right]
$$

where the subscript " $\phi "$ stands for the derivative with respect to $\phi$.
Now, suppose we have fixed the function $A(\phi)$. Then the class equation turns into a joint linear second-order 
ODE with unknown functions $\Lambda(\phi)$ and $\hat{\Xi}(\phi)$. This ODE thus is, in fact, the constraint for $\Lambda$ and $\hat{\Xi}$ which ensures the internal consistency of the theory. Therefore, with any given $A(\phi)$ one can associate the appropriate class of integrability given by a self-consistent $\{\Lambda, \hat{\Xi}\}$ pair. Thus, the space of all possible coupling functions becomes "inhomogeneous" as it can be divided according to the class structure. Theories belonging to a class are equivalent in a sense that their appropriate ground-state solutions possess the same dependence $A(\phi)$.

The next step is to find the most general class of equivalence which contains the static p-brane solutions related to $\mathrm{M}$ theory and possessing a SUSY limit, i.e., reducing to BPS states at some values of their parameters [18].
The latter will also ensure that quantum corrections are taken into account because the BPS states which define the class are protected from them and thus keep the class unchanged. The characteristic function $A(\phi)$ for this class is given by eq. (7) from ref. [16], and for a 0-brane reduces to

$$
A(\phi)=\frac{2 \varsigma}{a \tilde{p}} \phi+\frac{2}{\tilde{p}-1} \ln \kappa-\frac{2}{\tilde{p}-1} \ln \left(e^{\frac{\Delta \varsigma \phi}{2 a}}-1\right),
$$

where $\kappa$ is the integration constant which sets the brane's mass scale. Then we substitute it into the class equation above and obtain:

$$
\hat{\Xi}^{\prime \prime}(\phi)+\frac{4 \eta}{\phi_{1}} \hat{\Xi}^{\prime}(\phi)-\frac{2(\tilde{p}-1)}{\tilde{p}} \hat{\Xi}(\phi)+\kappa^{\frac{2 \tilde{p}}{\tilde{p}-1}} f(\phi)\left[V^{\prime \prime}(\phi)-\frac{\tilde{p} \operatorname{coth}\left(\frac{\phi}{\phi_{1}}\right)-\eta(\tilde{p}-2)}{\phi_{1}(\tilde{p}-1) / 2} V^{\prime}(\phi)+\frac{2}{\tilde{p}} V(\phi)\right]=0,
$$

where $f(\phi)$ is some function whose explicit form is not important here. Finally, as long as an action functional and hence coupling functions must be independent from the mass scale parameter $\kappa$ (see ref. [16], Sec. III) we can separate the coupling equations.
[1] J. Nishimura and F. Sugino, "Dynamical generation of four-dimensional space-time in the IIB matrix model," JHEP 05 (2002) 001.

[2] J. Nishimura, "Exactly solvable matrix models for the dynamical generation of space-time in superstring theory," Phys. Rev. D65 (2002) 105012.

[3] P. Bialas, et al, "Large N limit of the IKKT matrix model," Nucl. Phys. B592 (2001) 391.

[4] J. Ambjørn, J. Jurkiewicz and R. Loll, "Emergence of a 4D world from causal quantum gravity," Phys. Rev. Lett. 93, 131301 (2004); "Spectral dimension of the Universe," Phys. Rev. Lett. 95, 171301 (2005).

[5] Z. C. Wu, "Quantum Kaluza-Klein cosmologies. V," Gen. Rel. Grav. 34, 1121 (2002); "Dimensionality in the Freund-Rubin cosmology," Phys. Lett. B 585, 6 (2004).

[6] T. Damour and A. M. Polyakov, "The String dilaton and a least coupling principle," Nucl. Phys. B 423, 532 (1994).

[7] G. W. Gibbons and K. Maeda, "Black holes and membranes in higher dimensional theories with dilaton," Nucl. Phys. B 298, 741 (1988).

[8] R. Penrose, "Gravitational Collapse And SpaceTime Singularities," Phys. Rev. Lett. 14, 57 (1965); S. W. Hawking and R. Penrose, "The Singularities Of Gravitational Collapse And Cosmology," Proc. Roy. Soc. Lond. A 314, 529 (1970).

[9] R. Penrose, "Gravitational Collapse: The Role Of General Relativity," Riv. Nuovo Cim. 1, 252 (1969); Gen. Rel. Grav. 34, 1141 (2002).

[10] R. M. Wald, "Gravitational collapse and cosmic censorship," gr-qc/9710068

[11] P. R. Brady, I. G. Moss and R. C. Myers, "Cosmic Cen- sorship: As Strong As Ever," Phys. Rev. Lett. 80, 3432 (1998).

[12] K. G. Zloshchastiev, "Coexistence of black holes and a long-range scalar field in cosmology," Phys. Rev. Lett. 94, 121101 (2005).

[13] K. S. Thorne, R. H. Price and D. A. MacDonald (Eds.) Black holes: The membrane paradigm (Yale Univ. Press, New Haven, 1986); I. D. Novikov and V. P. Frolov, Physics of black holes (Kluwer, Dordrecht, 1989).

[14] J. D. Bekenstein, "Transcendence of the law of baryonnumber conservation in black hole physics," Phys. Rev. Lett. 28, 452 (1972); D. Sudarsky, "A simple proof of a no hair theorem in Einstein Higgs theory," Class. Quant. Grav. 12, 579 (1995).

[15] A. Strominger and C. Vafa, "Microscopic origin of Bekenstein-Hawking entropy," Phys. Lett. B 379, 99 (1996).

[16] K. G. Zloshchastiev, "Generic approach to dimensional reduction and selection principle for low-energy limit of M theory," hep-th/0512128 (v.3+).

[17] K. G. Zloshchastiev, "Core structure and exactly solvable models in dilaton gravity coupled to Maxwell and antisymmetric tensor fields," Phys. Lett. B 527, 215 (2002).

[18] K. S. Stelle, "BPS branes in supergravity," hep-th/9803116

[19] P. Van Nieuwenhuizen, "Supergravity," Phys. Rept. 68, 189 (1981).

[20] M.A. Abramowitz and I.A. Stegun (Eds.) Handbook of mathematical functions (Dover, NY, 1972).

[21] C. M. Hull and P. K. Townsend, "Unity of superstring dualities," Nucl. Phys. B 438, 109 (1995). 